\title{
ALPPS Offers a Better Chance of Complete Resection in Patients with Primarily Unresectable Liver Tumors. Results of a Multicentre Analysis: Reply
}

\author{
Erik Schadde • Roberto Hernandez-Alejandro • \\ Hauke Lang • Eduardo de Santibaňes • \\ Pierre-Alain Clavien
}

Published online: 6 March 2015

(C) Société Internationale de Chirurgie 2015

We read with interest the letter by Rohatgi et al. concerning our study comparing ALPPS with PVE/PVL in a population with mixed liver tumors [1]. Our study does not focus only on colorectal liver metastases, as is suggested in the letter's title. We studied a mixed population with different tumor etiologies. The authors main concern is the increased morbidity and mortality associated with ALPPS. They also take issues with a lack of adjustment for confounders in comparing the groups. The authors do not agree with our conclusion that rapid tumor removal in ALPPS may be advantageous, citing the argument frequently raised by the opponents of this new procedure, that failure to reach the second stage in staged procedures for cancer is just an unavoidable unmasking of the natural history of the disease [2]. Ultimately, they disagree that there is an advantage to ALPPS at all.

This is a response to the letter to the editor available at doi:10.1007/ s00268-014-2726-5.

E. Schadde $\cdot$ P.-A. Clavien $(\bowtie)$

Department of Surgery and Transplantation, Swiss HPB Centre, University Hospital Zurich, Rämistrasse. 100, 8091 Zurich,

Switzerland

e-mail: clavien@access.uzh.ch

R. Hernandez-Alejandro

Division of HPB Surgery, Department of Surgery, Western

University, London, ON, Canada

H. Lang

Department of Visceral Surgery and Transplantation, University of Mainz, Mainz, Germany

E. de Santibaňes

Division of HPB Surgery and Liver Transplant Unit, Department of Surgery, Italian Hospital, Buenos Aires, Argentina
We acknowledge-as we do in our paper-that ALPPS is associated with a higher complication rate than conventional approaches, and that this remains a concern despite the lack of statistical significance. As we are stressing in the paper, the study was not powered to detect differences in complications, but to test the hypothesis that makes ALPPS so alluring to many surgeons across the world: that ALPPS makes complete tumor removal feasible in a higher number of patients than conventional approaches to staged resection and volume enhancement. Despite the good news that ALPPS enabled complete tumor removal in basically all patients in our experience, we drew careful conclusions from the higher complication rate (see Table 6: Recommendations for ALPPS). We analyzed the association between ALPPS and complications in a subsequent analysis of the International ALPPS registry and found that age and indications other than colorectal metastases were associated with these complications [3]. In the early experience, which is reflected in our paper, the operation might have been used with too much enthusiasm as a "magic bullet" for a wide variety of tumors, often primary liver tumors with cholestasis, in older patients with extensive disease burden.

While we acknowledge the trend of a difference between a median ASA scores between the ALPPS and the PVE/PVL group, we ultimately decided not to include ASA score among the confounders. Due to the small cohort size, we opted for an adequate number of confounders for adjustment ( 5 confounders). We chose age (1) and gender (2), both of which are usually included in a multivariate analysis, additionally type of tumor (3), parenchymal quality (4), and starting future remnant volume (5), which are of clinical importance for the comparison of these two procedure types. Differently from the authors of this letter, we actually felt that the ALPPS group was rather at a 
disadvantage as compared to the PVE/PVL group as far as the severity of tumor burden in the liver was concerned: frequently the tumor burden motivated surgeons to use ALPPS instead of conventional approaches. Such differences are, however, difficult to adjust for, since they are neither captured by number nor by the size of tumors, specifically in a mixed tumor population. To us, "type of tumor" was the most important confounder that had to be corrected when looking at outcomes.

We also observed, as did the authors of this letter, that $11 \%(9 / 83)$ of patient did not undergo stage two in the PVE/PVL group, because they developed systemic progression, while all (48/48) ALPPS patients proceeded to stage two resection. However, we disagree with the authors that these $11 \%$ of patients in the PVE/PVL group would have had no benefit from ALPPS. Because the majority of patients with liver tumors die from the liver involvement, the answer to the question who benefits can only be given by survival data (and hopefully in the future: quality of life data) for separate tumor etiologies. We recently presented a $41 \%$ disease-free survival at 2 years after ALPPS from the International Registry [3], a good result for ALPPS when compared to two-stage hepatectomies for CLRM [4]. Our point in the comparative study [1] is that the advantage of surgical feasibility of resection in ALPPS does not translate into an increased recurrence rate, as has been postulated by some [2]. The argument that patients with recurrence between stages in PVE/PVL would not profit from rapid resection of their liver disease is treacherous since of course conventional staged approaches with longer waiting intervals will ultimately result in recurrence with prolonged waiting time. We maintain our conclusion that only a randomized prospective design can settle this controversy.

It is very well possible that, in the authors experience, very few patients fail the conventional staged approach in PVE and PVL. Like the authors, we are convinced that, specifically in CLRM, a careful selection of patients based on their response to chemotherapy and other predictors will reduce the drop-out rate in these conventional staged approaches. This, however, might as well be the population that also benefits the most from ALPPS due to a lower complication rate. Careful selection of patients for surgery makes a difference for both types of procedures. When we analyzed the subgroup of patients younger than 60 years with CLRM, we found a mortality of $5.1 \%$ and a rate of complications $>$ IIIB of only $16 \%[3,5]$, which compares favorably with a recently published meta-analysis for twostage hepatectomies [6]. The question posed by the authors of this letter, what proportion and kind of patient would really benefit from an ALPPS approach, will remain a question we have to study empirically. We invite the contributors of the International Registry (www.alpps.net) to help us to clarify this in the future.

\section{References}

1. Schadde E, Ardiles V, Slankamenac K et al (2014) ALPPS offers a better chance of complete resection in patients with primarily unresectable liver tumors compared with conventional-staged hepatectomies: results of a multicenter analysis. World J Surg 38(6):1510-1519. doi:10.1007/s00268-014-2513-3

2. Aloia TA, Vauthey JN (2012) Associating liver partition and portal vein ligation for staged hepatectomy (ALPPS): what is gained and what is lost? Ann Surg 256(3):e9 Author reply e16-9

3. Schadde E, Ardiles V, Robles-Campos R et al (2014) Early survival and safety of ALPPS-first report of the International ALPPS registry. Ann Surg 260(5):829-838

4. Brouquet A, Abdalla EK, Kopetz S et al (2011) High survival rate after two-stage resection of advanced colorectal liver metastases: response-based selection and complete resection define outcome. J Clin Oncol 29(8):1083-1090

5. Clavien PA, Barkun J, de Oliveira ML et al (2009) The ClavienDindo classification of surgical complications: five-year experience. Ann Surg 250(2):187-196

6. Lam VW, Laurence JM, Johnston E et al (2013) A systematic review of two-stage hepatectomy in patients with initially unresectable colorectal liver metastases. HPB (Oxford) 15(7):483-491 